\title{
Refractive index matched PIV experiments and CFD simulations of mixing in a complex dynamic geometry
}

Fenglei Huang ${ }^{a, b}$, Peng Chen ${ }^{a, b}$, Junhao Wang ${ }^{a, b}$, Zhipeng Li $^{a, b, *}$, Zhengming Gao ${ }^{a, b, *}$, Jos J. Derksen $^{c}$

${ }^{a}$ Beijing Advanced Innovation Center for Soft Matter Science and Engineering, Beijing University of Chemical Technology, Beijing 100029, China

${ }^{\mathrm{b}}$ State Key Laboratory of Chemical Resource Engineering, School of Chemical Engineering, Beijing University of Chemical Technology, Beijing 100029, China

${ }^{c}$ School of Engineering, University of Aberdeen, Aberdeen AB24 3UE, UK

* Corresponding author. Tel.: +8610 64418267; fax: +8610 64449862. E-mail address:

lizp@mail.buct.edu.cn (Zhipeng Li), gaozm@mail.buct.edu.cn (Zhengming Gao). 


\begin{abstract}
The flow fields under laminar conditions in two typical regions of a cavity transfer dynamic mixer consisting of an inner rotor and outer stator were visualized by refractive index (RI) matched particle image velocimetry (PIV) experiments. The RI of the working liquids and the transparent solid parts of rotor and stator were matched to allow for unobstructed optical access. The flow fields were predicted by using computational fluid dynamics (CFD) simulations including models for species transport. Various flow patterns in the dynamic mixer are discussed. The simulated flow fields in the two investigated regions agree well with the experimental data. The effect of gap width between rotor and stator $(\sigma)$ on the scalar mixing was evaluated, and smaller $\sigma$ will achieve better mixing because of the enhanced shearing and higher energy consumption.
\end{abstract}




\section{Introduction}

Mixing processes have been extensively researched for several decades, because of their relevance in many industrial applications. In many cases mixing takes place under turbulent flow conditions. Highly viscous liquids are, however, usually mixed at laminar conditions in areas such as rubber and plastics processing. ${ }^{1-3}$ Ottino and Chella ${ }^{4}$ found that efficient mixing can be obtained by repetitive stretching, folding, reorienting and stacking of the material in these laminar cases. A variant of this principle is applied in a dynamic mixer called Cavity Transfer Mixer (CTM), where mixing is achieved by smart shuffling of material between the many cavities the device is composed of. The CTM was invented in the 1980s by Gale at Rapra Technology Limited in order to improve distributive mixing of high viscosity fluids, and it is usually applied as an add-on unit for extruders. ${ }^{5}$ A dynamic mixer was developed by Jiang et al. to homogeneously mix micro/nano-inorganic particles in polymer melts. ${ }^{6}$ It is able to deal with a wide range of particle concentrations in their industrial applications. ${ }^{6}$ A CTM usually consists of two parts, a rotor and a stator. Each part has multiple rows of hemispherical cavities, as shown in Figure 1. The relative motion of rotor and stator continuously changes the relative position of the hemispherical cavities so that the liquid experiences a continuously changing flow geometry. The mixer generates a complex helical flow. There is a finite gap width between the rotor and the stator. The gap region is very small compared to the overall size of the entire dynamic mixer, but the high shear concentrated in this region might have significant effects on the mixing performance.

It is difficult to visualize the flow and mixing characteristics in a dynamic mixer based on laser-based optical measurement techniques because of its complicated and unsteady geometry. ${ }^{7}$ Most of the existing studies on dynamic mixers were conducted using computational fluid dynamics (CFD). The first three-dimensional simulation of a dynamic mixer was performed to characterize the flow fields based on 
the finite element method, and the potential use of the mixer for dispersive mixing was proposed by Wang and Manas-Zloczower. ${ }^{8}$ Grosso et al. ${ }^{9}$ conducted comprehensive three-dimensional simulations to assess the mixing processes in a CTM. Their work found that the number of cavities per row and the dimensionless number $k$ defined as the ratio between the axial and the tangential velocity of the fluid at the inlet are the two critical factors for the performance of the dynamic mixer. Many studies simplified dynamic mixers into two-dimensional approximations and conducted corresponding simulations. ${ }^{10-13}$ Woering et al. proposed a more specific and quantitative mixing analysis by investigating the interactions between two rectangular cavities. ${ }^{10}$ Recently, Grosso et al. captured stretching-and-folding mixing actions inside the device and assessed the impact of geometrical and operational variables by a two-dimensional mixer model. ${ }^{11}$

Optical measurement techniques with laser light sources such as Particle Image Velocimetry (PIV) have been used in various areas to investigate single-phase flow, two miscible fluid flow and dilute two-phase flow. $^{14-18}$ Refractive index matching (RIM) is a technique commonly used to overcome optical inaccessibility of multiphase systems and complex geometries. ${ }^{19-20} \mathrm{H}$. Mazhar et al. used a $60 \%$ w/w Sodium Iodide $(\mathrm{NaI})$ aqueous solution as the working fluid to match the refractive index of their acrylic test section and obtained the flow fields in a dual S-shape $90^{\circ}$ bend by PIV. ${ }^{21}$ Jaffer et al. used PIV to obtain the flow fields in kneading disc region of twin-screw extruder, the $1.0 \mathrm{~Pa} \cdot \mathrm{s}$ mineral oil was used as the working fluids to match the refractive index of the transparent extruder. ${ }^{22}$ The simulated results are in fair agreement with experimental results. The paper shows the tremendous promise for applying PIV to complex dynamic mixing systems containing high-viscosity fluids. In the current work, RI matched PIV was applied to a complexly shaped system with strongly curved walls consisting of a static and a rotating element. A highly viscous maltose aqueous solution $(\mu=20 \mathrm{~Pa} \cdot \mathrm{s}$ at $21 \square)$ with almost the same refractive 
index as the material of the mixer was selected as working fluid to provide a viable environment for detailed PIV experiments.

In our previous work, ${ }^{7}$ the dynamic mixer geometry was simplified to a three-dimensional lid-driven cavity flow model to predict the mixing process of two miscible fluids. The influences of viscosity and density difference of the two miscible fluids on the mixing process were evaluated. The dimensionless group $\frac{A r}{R e}$ (Archimedes number over Reynolds number) was proposed to characterize the mixing process in the lid-driven cavity. ${ }^{7}$ In the current study, $\frac{A r}{R e}$ has been applied to characterize the mixing process of two liquids in the dynamic mixer with complex geometry for the first time.

In the first place the aim of this paper is to show the feasibility of PIV experiments in a complex structure with strongly curved walls and relative motion between rotor and stator. Secondly, by comparing simulated flow fields in the dynamic mixer with the experimental data, we validate our simulation results. In the third place, we investigated the effect of gap width on the mixing performance based on the verified simulation methods and models. Finally, the dimensionless group $\frac{A r}{R e}-$ which proved useful in our previous work on a lid-driven cavity - was applied to the dynamic mixer.

This paper is organized as follows: in the next section, the experimental setup is discussed, including the flow system and PIV experimental setup. Then, the numerical approaches that we used in this work are briefly summarized with references. In the subsequent Result and Discussion section, we first present various flow patterns in this complex flow system. Secondly, we compare the simulated velocity profiles with the PIV experimental data. Thirdly, we study the effects of gap width and the dimensionless group $\frac{A r}{R e}$ on mixing between two fluids in the dynamic mixer and use the coefficient of variation (COV) for characterizing mixing efficiency. The final section reiterates the main conclusions and suggests directions for future work. 


\section{Experiments}

\subsection{Flow system}

Figure 1(a) shows the experimental setup, including the dynamic mixer made of Polymethyl methacrylate (PMMA). A computer-controlled stepping motor (CNYOHO, China) drives the rotor at a given speed $N=1 \mathrm{rev} / \mathrm{min}$. Fluctuations of $N$ were within $\pm 1 \%$. There are three rows (in circumferential direction) of cavities in the stator and two rows of cavities in the rotor. There are two inlets at the left of the dynamic mixer, Inlet 1 for Liquid 1 and Inlet 2 for Liquid 2. The volumetric flow rates of Inlet 1 and Inlet 2 are $48 \mathrm{ml} / \mathrm{min}$ and $12 \mathrm{ml} / \mathrm{min}$, respectively. The outlet is at the right bottom of the dynamic mixer. Dimensionless time is measured in rotor revolutions $(t N)$. The diameter of the rotor is $D=84 \mathrm{~mm}$, and the diameter of the cavities in the rotor and stator is $d=40 \mathrm{~mm}$. There is a gap between rotor and stator, and the gap width $(\sigma)$ is $0.5 \mathrm{~mm}$, as marked in Figure 1(c). As shown in Figure 1(c), five sections (at $x / d=0.34$, $x / d=1.46, x / d=2.59, x / d=0.50$ and $x / d=1.06$ ) have been selected to discuss the results in this paper. All cavities in a row are numbered from 1 to 6 in a clockwise direction, as shown in Figure 1(b). In the experiments, the velocity magnitude due to the pressure driven flow in Section 4 was $0.0002 \mathrm{~m} / \mathrm{s}$ which is about $4.5 \%$ of the velocity induced by rotation $(\pi N D=0.0044 \mathrm{~m} / \mathrm{s})$. 


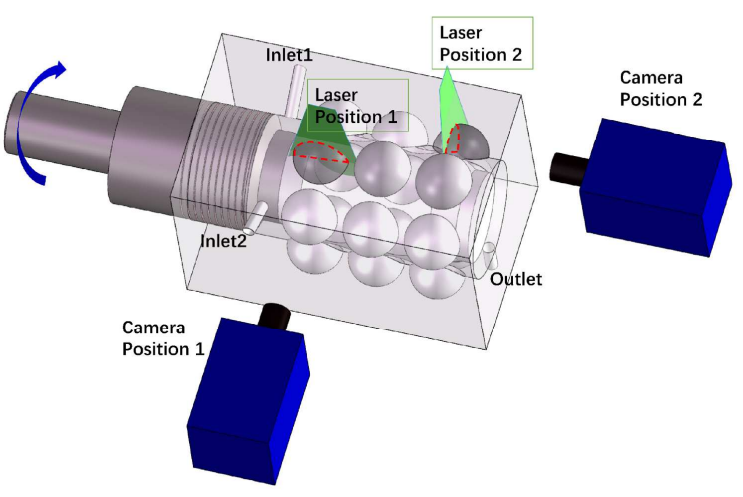

(a)

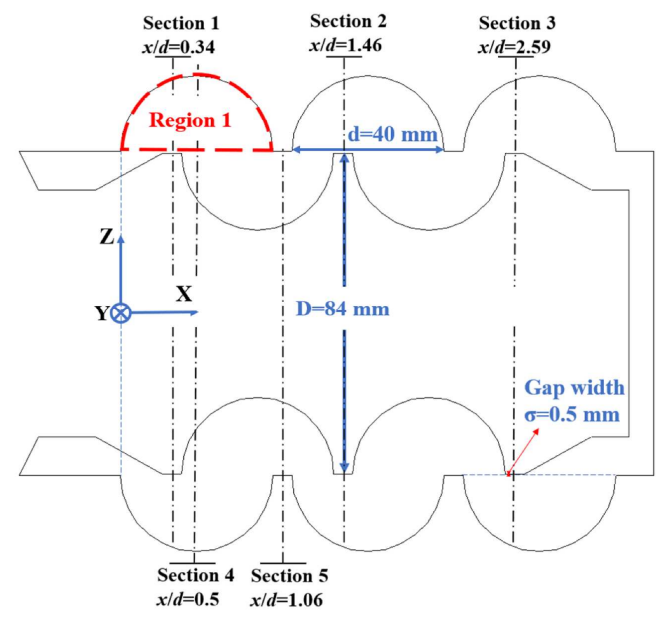

(c)

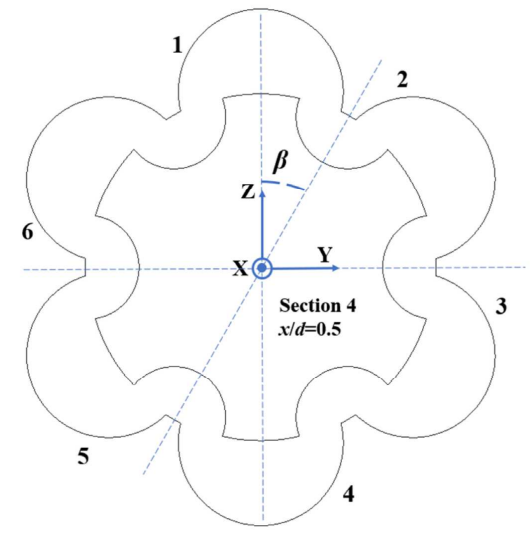

(b)

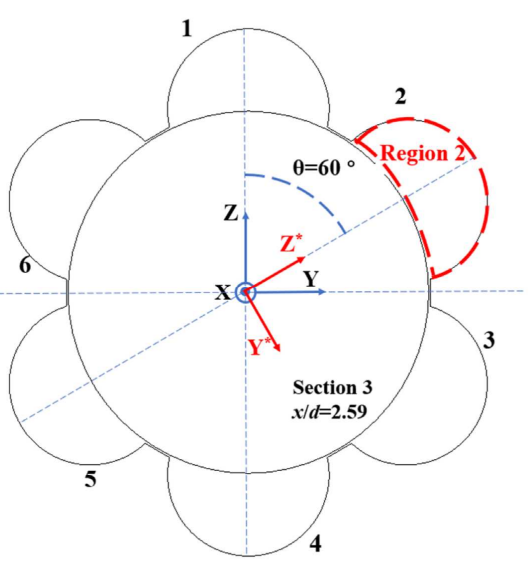

(d)

Figure 1. (a) Schematic experimental setup with three rows of stator cavities and two rows of rotor cavities, two typical regions and corresponding camera and laser positions for PIV experiments. (b) Relative angle between rotor and stator. (c) Plane $y / d=0$; The coordinate system, parameters of the dynamic mixer and five selected sections for discussions in this paper. (d) Specific coordinate system for Region 2 at Section 3.

To match the refractive index of the PMMA ( $\mathrm{n}=1.487)$, a maltose aqueous solution $(77.2 \mathrm{wt} \%, \rho=1394$ $\mathrm{kg} / \mathrm{m}^{3}, \mathrm{n}=1.487, \mu=20 \mathrm{~Pa} \cdot \mathrm{s}$ at $21 \square$ ) was made as Liquid 1 and Liquid 2 . The dynamic viscosity was measured with a MARS40 Rheometer (Haake, Germany) and the refractive indices with a WAY-2W Abbe refractometer (Shanghai INESA Instrument, China). Images of a ruler in a cavity of a dynamic mixer made by PMMA with air, deionized water and the maltose aqueous solution are shown in Figure 2 . The actual width of the ruler is denoted as $W r$. The dimensions $W_{l}$ and $W_{2}$ are clearly smaller than the real width $W r$, and the shape of the ruler has visible optical distortions in Figure 2(a) \&(b). The width $W_{3}$ is 
almost the same with $W r$, and the shape of ruler in Figure 2(c) is not distorted and clear. In this paper, we use this refractive index matched system with PMMA and the maltose aqueous solution for the dynamic mixer and the working fluid respectively.

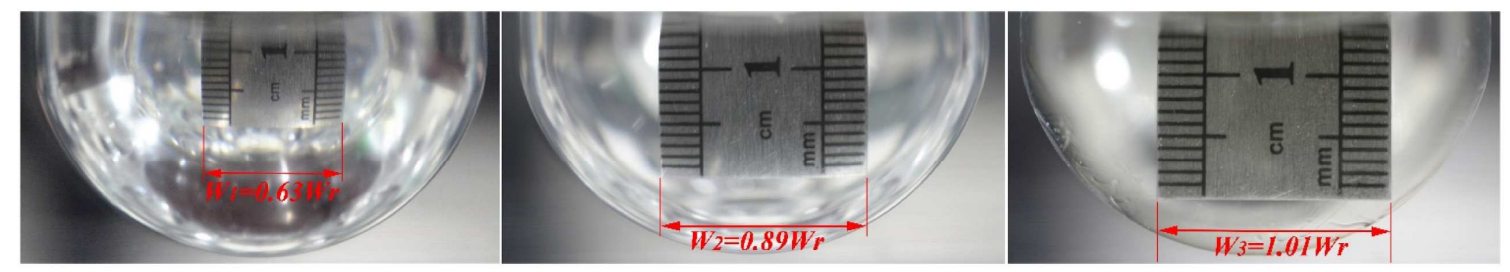

(a)

(b)

(c)

Figure 2. Images of a ruler in a cavity of the dynamic mixer made by PMMA ( $n=1.487)$ filled with (a) air ( $\mathrm{n}=1.000)$; (b) deionized water $(\mathrm{n}=1.333)$; (c) the maltose aqueous solution used in this work $(\mathrm{n}=1.487) . \mathrm{Wr}$ is the real width of the ruler.

The dynamic mixer was filled with Liquid 1 in the initial state. In the experiments, the Reynolds number $\operatorname{Re}=\frac{\rho_{1} N D^{2}}{\mu_{1}}\left(\mu_{1}\right.$ the viscosity of Liquid $1, \rho_{1}$ the density of Liquid 1$)$ is 0.008 . The Archimedes number is defined as $A r=\frac{g D^{3} \rho_{1}\left(\rho_{1}-\rho_{2}\right)}{\mu_{1}{ }^{2}}$ ( $g$ is gravitational acceleration in the direction of negative $z, \rho_{2}$ is the density of Liquid 2) to investigate the effects of density difference on the mixing process of the two miscible fluids. In the experiments $A r$ is equal to zero due to the same density but non-zero $A r$ has been considered in the simulations. Another dimensionless number (Schmidt number) $S c=\frac{\mu}{\rho D_{m}}$ is also considered as an essential variable that affects the mixing performance of miscible fluids. The self-diffusion coefficient of the maltose aqueous solution used in this paper is about $10^{-11} \mathrm{~m}^{2} / \mathrm{s},{ }^{23}$ which leads to $S c$ reaching a value of the order of $10^{9}$ for Liquid 1 as well as Liquid 2. The influence of mass diffusion on the mixing performance is expected to be negligible in this experiment because the Peclet number $(P e=S c R e)$ which determines whether the flow is controlled by convection or diffusion is much larger than 1 in all cases, such as when $R e=0.008, P e=1.1 \times 10^{7}$.

\subsection{PIV experiments}

The 2D-PIV system used in this paper is a commercial system from TSI (USA). It consists of a $532 \mathrm{~nm}$ 
$200 \mathrm{~mJ} \mathrm{Nd:YAG} \mathrm{dual} \mathrm{pulse} \mathrm{laser} \mathrm{(Beamtech,} \mathrm{China),} \mathrm{spherical} \mathrm{and} \mathrm{cylindrical} \mathrm{lenses} \mathrm{which} \mathrm{transform} \mathrm{a}$ laser beam into a laser sheet with thickness of $1 \mathrm{~mm}$ at the measurement plane, a $4008 \times 2672$ pixels charge-coupled device (CCD) camera (TSI, USA), a synchronizer (TSI, USA), and the Insight 4G software. Hollow spherical glass particles (TSI, USA) with density of $1500 \mathrm{~kg} / \mathrm{m}^{3}$ and averaged diameters of about $10 \mu \mathrm{m}$ were used as PIV tracer particles. The transistor-transistor logic (TTL) signals generated by the angle-encoder were used to synchronize the relative position between rotor and stator, the laser pulses, and the image capture. The relative angle between rotor and stator is defined as $\beta$ as shown in Figure 1(b). We only captured the flow fields at $\beta=0^{\circ}$ in the PIV experiments.

In Figure 1, the coordinate system is defined and marked in blue. The velocities in the three Cartesian directions $(x, y, z)$ are denoted by $u, v$, and $w$ respectively. Two typical regions were selected to capture the flow with PIV, as shown in Figure 1(c) and Figure 1(d). Region 1 is in stator cavity 1 in the first row in the $y / d=0$ plane, and Region 2 is in stator cavity 2 in Section 3. For a good view of Region 2, the coordinate system is rotated by $\theta=60^{\circ}$ in the clockwise direction and presented as $x^{*}, y^{*}, z^{*}$ as shown in Figure 1(d). The raw PIV images were post-processed by Insight 4G software (TSI, USA). The minimum size of the interrogation windows was $64 \times 64$ pixels $^{2}$, and the grid step size was $32 \times 32$ pixels $^{2}$. The image resolution was 12.69 and $14.19 \mu \mathrm{m} /$ pixel for Region 1 and Region 2 respectively, thus the velocity vector resolution was one vector per $0.40 \mathrm{~mm}$ and $0.45 \mathrm{~mm}$ (as compared to the cavity diameter of $d=40 \mathrm{~mm}$ ). The time interval between the two laser pulses - that together generate a single PIV velocity vector field $\Delta t$ was determined as $132000 \mu$ s and $56000 \mu$ s for Region 1 and Region 2 to ensure that the maximum in-plane and out-of-plane displacements of the tracer particles were smaller than one-quarter of the interrogation windows size and of the thickness of the laser sheet. ${ }^{24-25}$ One hundred pairs of raw PIV images were used for the averaged flow fields for each region. 


\section{Simulations}

\subsection{Species transport model}

Simulations of flows of multi-component Newtonian fluids are based on the numerical solution of the continuity and Navier-Stokes equations:

$$
\begin{gathered}
\frac{\partial \rho}{\partial t}+\nabla \cdot(\rho \vec{u})=0 \\
\frac{\partial \rho \vec{u}}{\partial t}+\nabla \cdot(\rho \vec{u} \vec{u})=-\nabla p+\nabla \cdot(\mu \nabla \vec{u})+\rho \vec{g}
\end{gathered}
$$

where $\rho$ is the fluid density, $\vec{u}$ is the velocity, $p$ is the pressure, $\mu$ is the viscosity and $\vec{g}$ is the gravitational acceleration.

A species transport model was applied to simulate the mixing process of the two miscible high viscosity fluids in the dynamic mixer. The conservation equation of species $i$ is: ${ }^{26}$

$$
\frac{\partial\left(\rho f_{i}\right)}{\partial t}+\nabla \cdot\left(\rho f_{i} \vec{u}\right)=\nabla \cdot\left(\rho D_{m} \nabla f_{i}\right)
$$

where $D_{\mathrm{m}}$ is the mass diffusion coefficient, and $f_{i}$ is the local mass fraction of the species $i$. In the system, an equation in this form will be solved for $n-1$ species with $n$ the total number of fluid species. The $n_{\text {th }}$ mass fraction is calculated by one minus the sum of the $n-1$ solved mass fractions. In this paper, the conservation equation of Liquid 1 was solved, and the mass fraction of Liquid 2 was calculated by one minus the mass fraction of Liquid 1.

The effective viscosity of the mixture is calculated from the mass fraction and the viscosity of each liquid in the following linear way:

$$
\mu=f_{1} \mu_{1}+\left(1-f_{1}\right) \mu_{2}
$$

where $\mu_{1}$ and $\mu_{2}$ are the viscosities of Liquid 1 and 2 respectively, and $f_{1}$ is the mass fraction of Liquid 1.

The density of the mixture is calculated by means of the mass fraction and the density of each liquid 
as follows:

$$
\frac{1}{\rho}=\frac{f_{1}}{\rho_{1}}+\frac{\left(1-f_{1}\right)}{\rho_{2}}
$$

where $\rho_{1}$ and $\rho_{2}$ are the densities of Liquid 1 and Liquid 2 respectively.

\subsection{Numerical details}

Direct simulation was used to calculate the flow in the dynamic mixer because the largest Reynolds number of all simulation cases was 0.32 based on the commercial software package Fluent 19.1. The sliding mesh technique is used to simulate the relative motion between rotor and stator. At the initial state, the dynamic mixer was filled with Liquid 1. Liquid 2 was introduced through Inlet 2. The species transport model was selected to simulate the mixing of two miscible high viscosity fluids. The geometric configuration applied for the CFD simulations in this work was the same as that used for the PIV experiments. A commercial software ICEM was used to construct the geometry and the mesh. ${ }^{27}$ The robust octree-based algorithm in ICEM was applied to generate grids. About 6.0 million unstructured tetrahedron cells were employed for meshing the geometry. The size of the mesh was $1 \mathrm{~mm}$ for global dynamic mixer and $0.5 \mathrm{~mm}$ for the cavities. A grid sensitivity check has been carried out, and the results will be explained in Section 4.2. The sliding mesh was divided into two parts and at the sliding interface a conservative interpolation is used for both mass and momentum. The no-slip condition was applied to all solid wall boundaries. As for the temporal resolution, time step $\Delta t$ was set as $0.1 \mathrm{~s}$ to keep the Courant-Friedrichs-Levy number $\left(\frac{U \Delta t}{\Delta x}\right)$ less than $1 .^{28}$ The third-order MUSL scheme ${ }^{29-30}$ was used for the spatial discretization of the momentum equations and the bounded second-order implicit scheme for time advancement. Liu found that first order upwind scheme could produce more false diffusion than high order schemes. ${ }^{31}$ Therefore, the third-order MUSL scheme was used for spatial discretization of species transport equations. For each time step, convergence was achieved when the normalized residuals of the 
mass fraction became less than $10^{-7}$ and the normalized residuals of the continuity and momentum became less than $10^{-4}$. The simulations based on 6.0 million cells take about 24 hours for per revolution on a computer with two Intel Xeon 2650 CPUs.

\section{Result and discussion}

\subsection{Comparisons of simulations and experimental results}

Velocity fields at $\beta=0^{\circ}$ obtained by PIV in Region 1 are shown in the left panel of Figure 3 . As explained above, this is the average vector field based on 100 realizations of the flow all measured at the same rotor angle $\left(\beta=0^{\circ}\right)$. The simulated velocity field in Region 1 at moment $t N=1$ is presented in the right panel of Figure 3. As will be argued later in this paper, the simulations indicate that after one rotor revolution the flow has fully developed so that comparing a simulation snapshot at $t N=1$ with an average experimental result is justified. There is good agreement between experiment and simulation. There are some slight differences: for instance, the experimental velocities at the right-bottom corner of this cavity are smaller than the simulated results. To better visualize the discrepancy between experimental and simulated results in terms of fluid velocity, $u$ and $v$ profiles on a horizontal line $(z / d=1.2)$ are shown in Figure 4. Although there are some discrepancies such as a more negative $u$ in the simulations as compared to the experiment on the horizontal line from $x / d=0.5$ to $x / d=1.0$, the simulated results agree generally well with the experimental data. This gives us confidence to use simulated results for predicting the mixing

process of two miscible high viscosity fluids in this complex system. As also shown in Figure 4, the simulated velocity results with 6.0 million cells can be considered grid independent, given that the velocity profiles obtained with 10.0 million cells have good agreements with those of 6.0 million cells. 

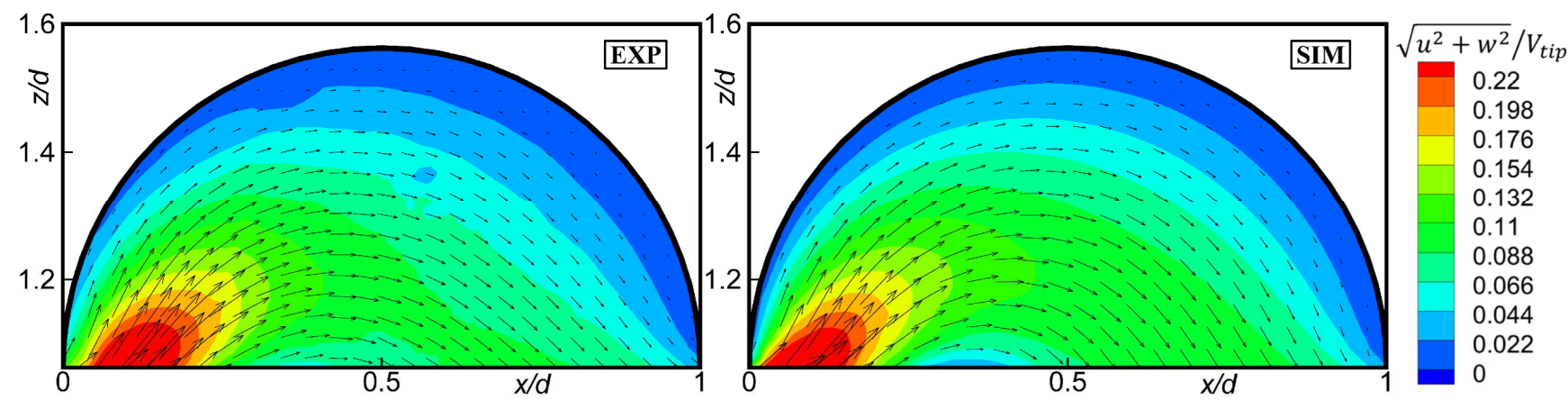

Figure 3. Averaged experimental velocity fields in Region 1 at $\beta=0^{\circ}$ (left column); Simulated velocity fields at $t N=1$ in Region 1 at $\beta=0^{\circ}$ (right column).
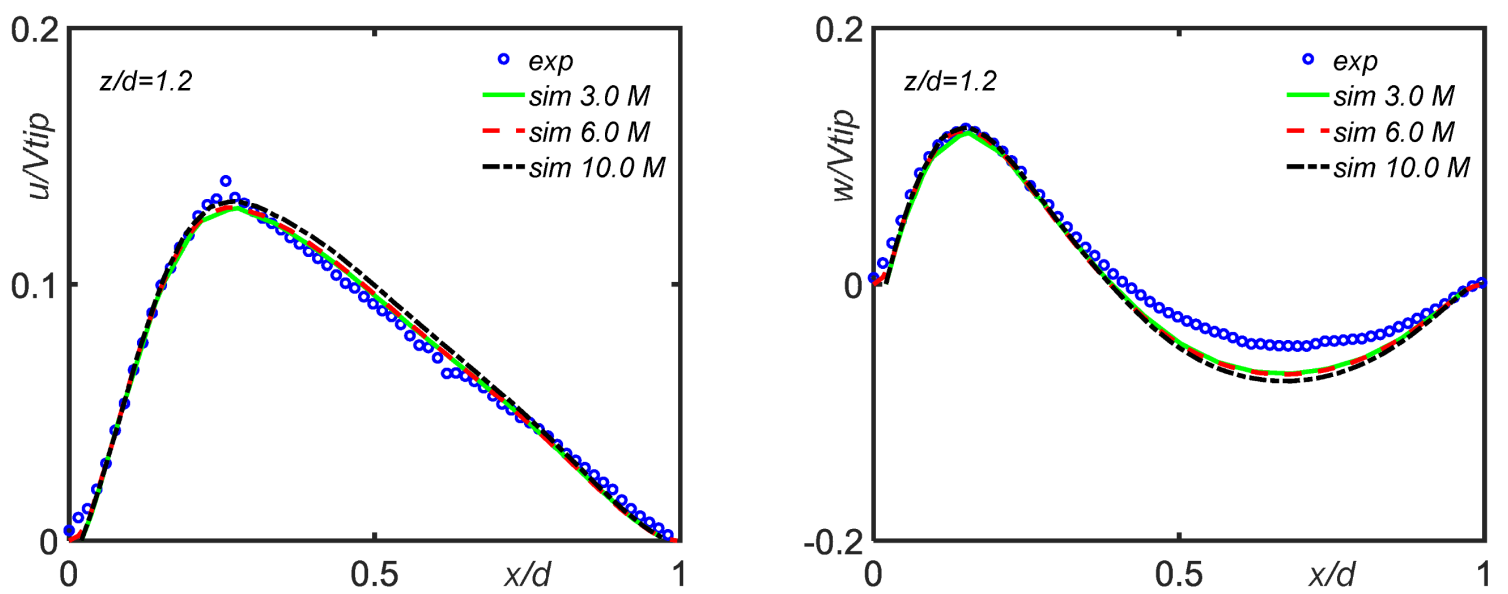

Figure 4. Experimental and simulated velocity profiles on a horizontal line $z / d=1.2$ in Region 1 at $\beta=0^{\circ}$. Simulated results with 3.0, 6.0, and 10.0 million cells are shown in each panel at $\beta=0^{\circ}$.

The instantaneous velocity profiles on the horizontal line $(z / d=1.2)$ in Region 1 at $t N=1 / 6,1$ and 5 are shown in Figure 5. The velocity components $u$ and $v$ on the horizontal line $(z / d=1.2)$ show that the simulated flow at $\beta=0^{\circ}$ is already fully developed after $t N=1$ because the result at $t N=1$ overlaps that at $t N=5$ in Figure 5. Therefore, the comparison of experiments and simulations are discussed based on the simulated results at $t N=1$. 

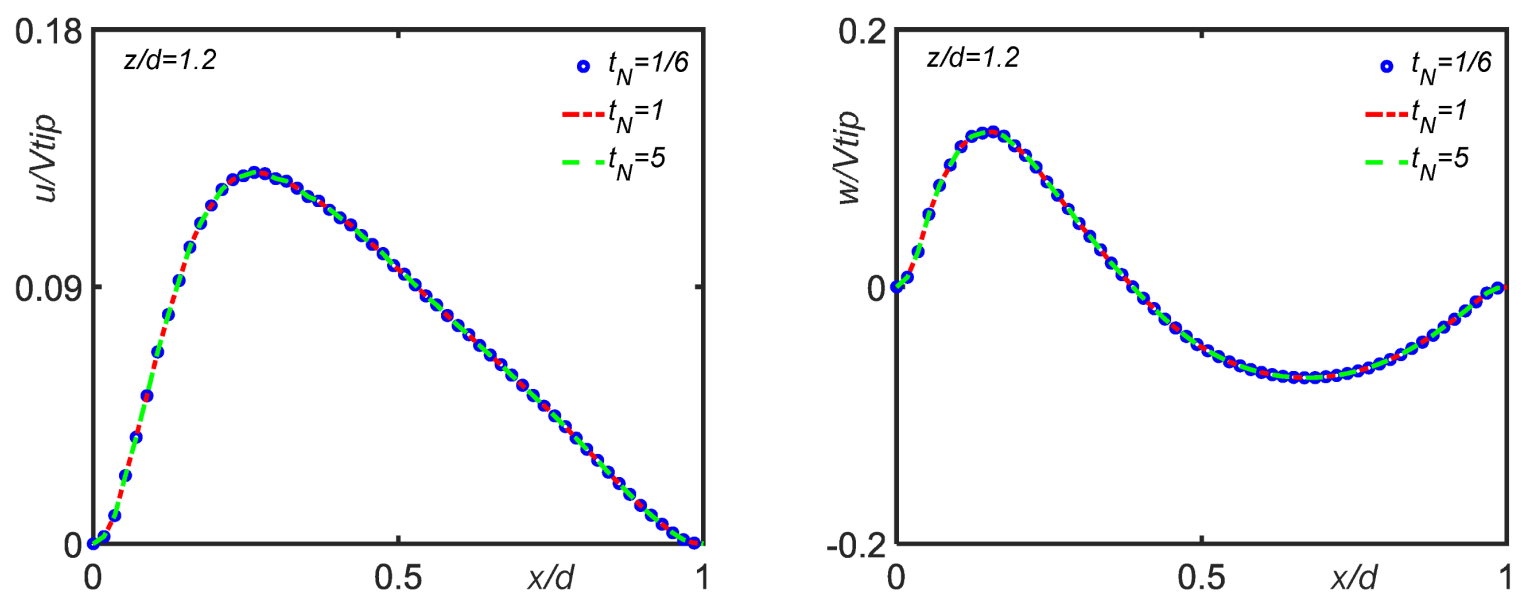

Figure 5. Instantaneous velocity profile $a t \beta=0^{\circ}$ on a horizontal line $z / d=1.2$ in Region 1 at $t N=1 / 6, t N=1$ and $t N=5$.

In order to further verify the accuracy of the numerical simulations, the average experimental flow fields (at $\beta=0^{\circ}$ ) obtained by PIV experiments in Region 2 are shown in Figure 6, left panel. The simulated velocity fields at $t N=1$ in Region 2 are in the right panel of Figure 6. From Figure 6, it is evident that the simulation can predict the flow pattern in the rotation direction very well. To allow for quantitative comparison of the experimental and simulated results, we present $u^{*}$ and $w^{*}$ velocity profiles on the horizontal line $z * / d=1.2$ in Figure 7 . In general, the simulated and experimental results along this line agree well with each other. As shown in Figure 7, the velocity profiles also confirm that our simulated velocity fields with 6.0 million cells are already grid insensitive.
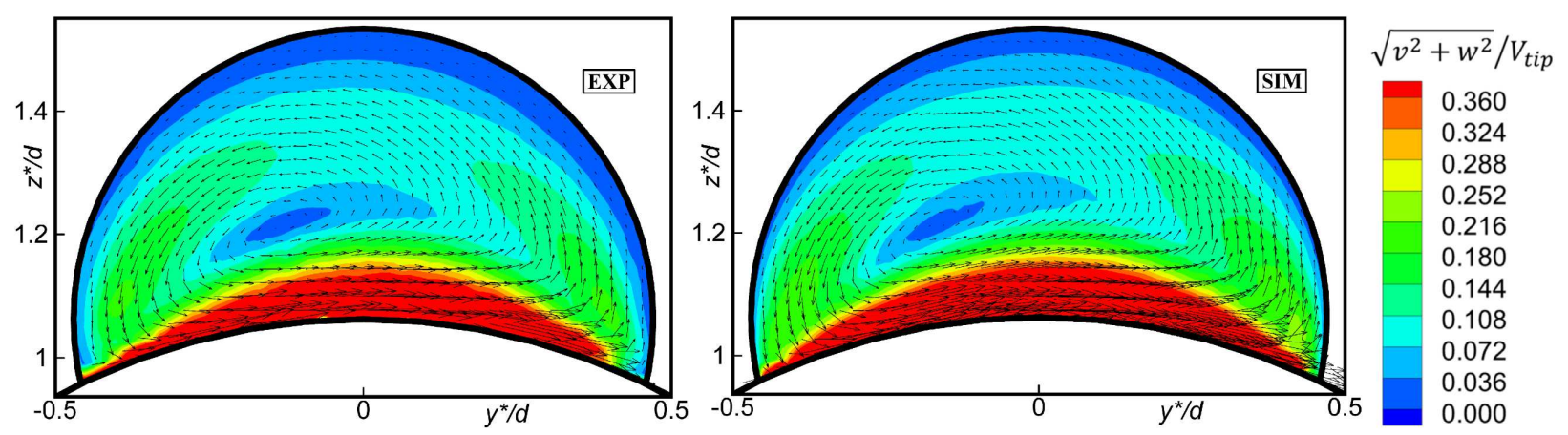

Figure 6. Averaged experimental velocity fields in Region 2 at $\beta=0^{\circ}$ (left column); Simulated velocity fields at $t N=1$ in Region 2 at $\beta=0^{\circ}$ (right column). 

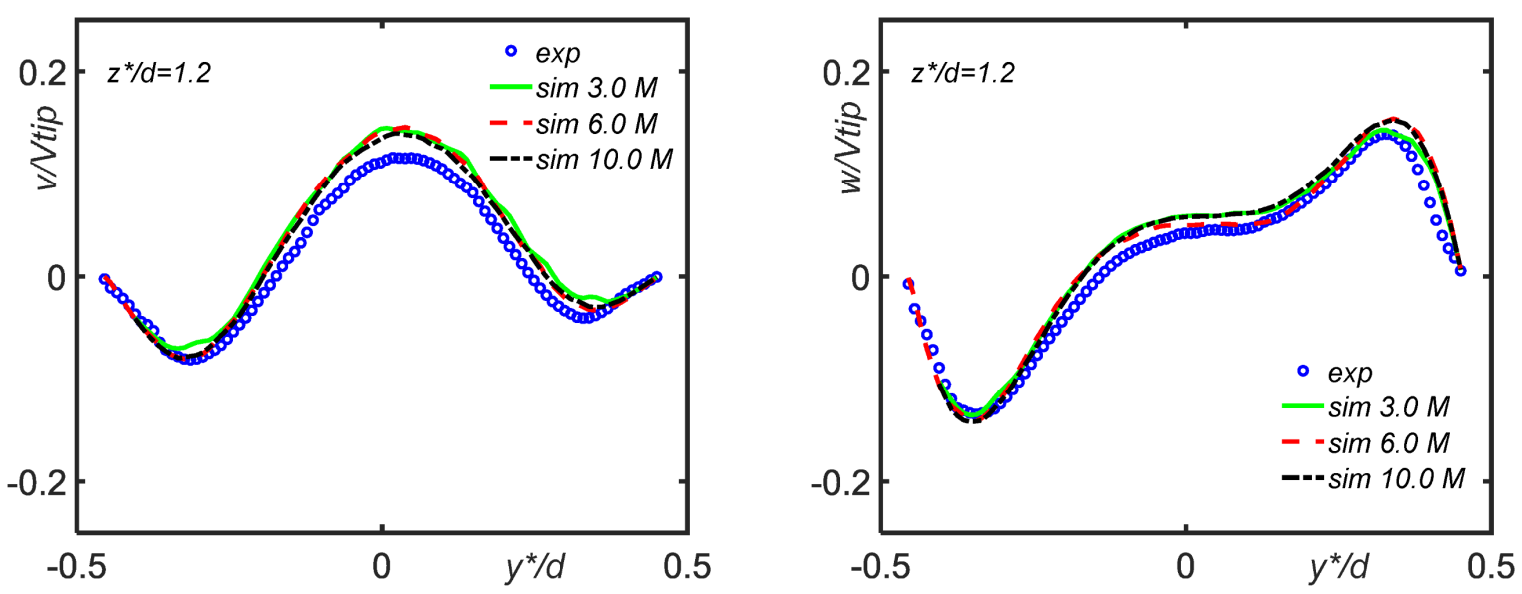

Figure 7. Experimental and simulated velocity profiles on a horizontal line $z^{*} / d=1.2$ in Region 2 at $\beta=0^{\circ}$. Simulated results with 3.0, 6.0, and 10.0 million cells $a t \beta=0^{\circ}$ are shown in each panel.

In summary, for two typical regions, one in the flow and one in the rotation directions, simulations have been validated by the experimental results. We conclude that simulations predict the flow fields of the mixing with two high viscosity miscible fluids in a dynamic mixer well. In the next section, various patterns in the flow and rotation directions in the dynamic mixer will be discussed.

\subsection{Flow fields in the dynamic mixer}

The flow in the dynamic mixer is complicated since various flow patterns are involved. There are six stator cavities and six rotor cavities in each row so that the flow is $60^{\circ}$ periodic. Four typical flow patterns in terms of velocity vectors and velocity magnitude contours in the $y / d=0$ plane in one period are shown in Figure 8 . The angle $\beta$ is the angle within a period (as defined in Figure $1 \mathrm{~b} ; 0^{\circ} \leq \beta \leq 60^{\circ}$ ). The overall flow is from left to right, driven by the pressure difference between the inlets and outlet. The outlet is at the bottom right corner of the panels of Figure 8. It shows relatively high velocities. On its way from inlets to outlet, the fluids undergo a series of contractions and expansions, resulting in repetitive stretching and folding which promotes mixing. As a result of the combined actions of rotor rotation and pressure driven flow, after injection the fluid first enters a stator cavity. It is then transferred to a rotor cavity, then to 
another stator cavity and so on. The way and rate of transfer from one cavity to the next depends on $\beta$ as can be appreciated from the panels in Figure 8 with the inset panels focusing on a central stator cavity (with red circumference) and the rotor cavities it is in contact with (green circumferences). Dependent on $\beta$, stator and rotor cavities overlap in different manners, resulting in a variety of flow paths for the fluid. When $\beta=0^{\circ}$ (Figure $8 \mathrm{a}$ ), the stator cavities and rotor cavities are aligned, with one stator cavity in contact with two rotor cavities (see the inset a1 of Figure 8). In the other panels in Figure 8, one stator cavity is in contact with three or four rotor cavities. This causes cross flow (i.e. flow in the tangential direction) not only in the rotor but also in the stator. In addition, narrow flow paths between rotor and stator can be observed. In Figure $8 \mathrm{~b}\left(\beta=15^{\circ}\right)$, there are reflux zones in the right part of the stator cavities. This flow pattern promotes folding, reorienting and stacking of the high viscosity liquids. At $\beta=30^{\circ}$ (Figure $8 \mathrm{c}$ ) there is no overlap between rotor and stator cavities in the $y / d=0$ plane. As a result, the flow velocities in the stator cavities are low in this plane. The configuration of cavities in Figure 8d is mirror-symmetric with that in Figure $8 \mathrm{~b}$. The flow in the symmetry plane (with $y / d=0$ ) is as shown in Figure $8 \mathrm{~d}$ is, however, markedly different from that in Figure $8 b$. This is due to the way liquid is fed to the mixer with Inlet 1 having a volumetric flow rate that is four times that of Inlet 2. In summary, the velocity fields in the flow direction are complex and strongly vary with the relative position of the rotor and stator. 



Figure 8. Simulated velocity field in $y / d=0$ plane. (a) At $t N=1, \beta=0^{\circ}$; (b) At $t N=\frac{25}{24}, \beta=15^{\circ}$; (c) At $t N=\frac{13}{12}, \beta=30^{\circ}$; (d) At $t N$ $=\frac{9}{8}, \beta=45^{\circ}$. The insets (a1, b1, c1 and d1) in each panel focus on a central stator cavity (circled red) and the rotor cavities it is in contact with (circled green) at its specific angle $\beta$. 

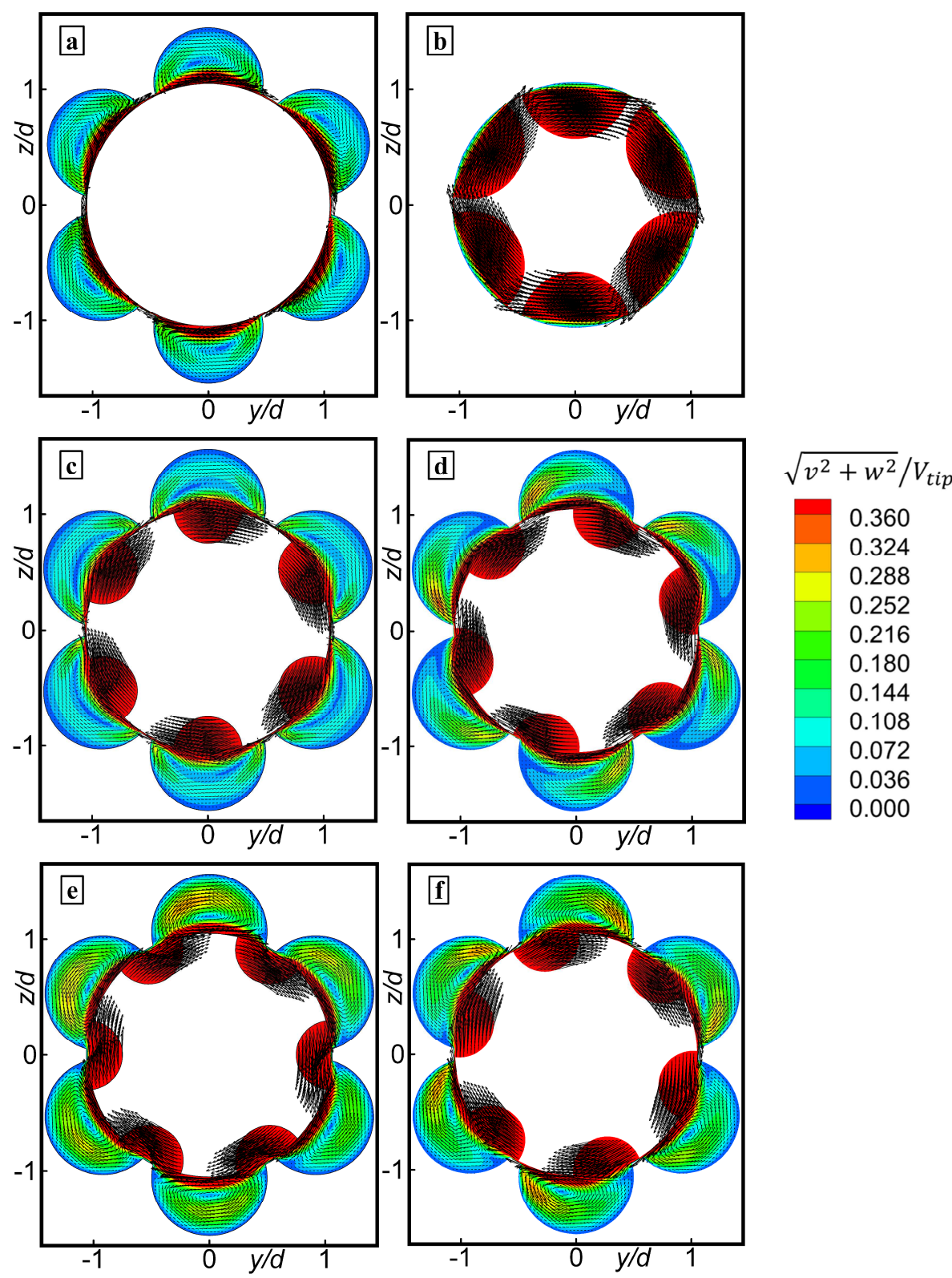

Figure 9. Simulated velocity field. (a) In Section 3 at $t N=1, \beta=0^{\circ}$; (b) In Section 5 at $t N=1, \beta=0^{\circ}$; (c) In Section 4 at $t N$ $=1, \beta=0^{\circ}$; (d) In Section 4 at $t N=\frac{25}{24}, \beta=15^{\circ}$;(e) In Section 4 at $t N=\frac{13}{12}, \beta=30^{\circ}$; (f) In Section 4 at $t N=\frac{9}{8}, \beta=45^{\circ}$.

In Figure 9 the simulated flow in circumferential cross sections has been visualized. In the stator cavities, the liquid is recirculating as a result of the shearing motion in the circumferential direction induced by either the rotor itself (as for example in Figure 9a), or by the combined action of the liquid in the rotor cavities and the rotor walls. This flow pattern is very similar to a lid-driven cavity flow. ${ }^{32-33}$ The location of the vortex center and the shape of the vortex in the stator cavities depends on how exactly it is 
being generated by the rotor and its cavities. Also in the rotor cavities, the liquid is strongly sheared while - at the same time - it moves with the rotor in the circumferential direction. It is also worthwhile pointing out that relatively high liquid velocities are encountered in the gap between rotor and stator, see e.g. Figure 9a.

In this section, the velocity fields with the various patterns in the flow and rotation directions in the dynamic mixer have been discussed. The combination of these velocity fields and flow patterns form the basis for mixing. In the next section, many sets of simulations will be discussed to assess the mixing performance of the dynamic mixer.

\subsection{Scalar Mixing in the dynamic mixer}

\subsubsection{The effect of gap width}

To investigate the effect of the gap width on mixing performance, three cases with different $\sigma(0.5$ $\mathrm{mm}, 1.5 \mathrm{~mm} 3.0 \mathrm{~mm}$ ) were conducted, where $\sigma=0.5 \mathrm{~mm}$ is the "default" value with which we studied verification and validation of the simulations in the previous section. Instantaneous concentration distributions of Case $1(\sigma=0.5 \mathrm{~mm})$ at $t N=10$ in different $y-z$ sections are shown in Figure 10(a). Liquid 2 flows from Section 1 to Section 3 and experiences repetitive stretching, folding and stacking in the dynamic mixer. The two liquids are still clearly segregated in Section 1 . When reaching Section 3, a significant degree of mixing has taken place. Levels of mixedness get somewhat worse with increasing gap width $\sigma$. Figures $10(\mathrm{~b})$ and $10(\mathrm{c})$ show results for $\sigma=1.5 \mathrm{~mm}$ and $\sigma=3.0 \mathrm{~mm}$ respectively. The differences as a result of gap width are most apparent in Section 2 where Liquid 2 concentration levels above 0.8 appear for the larger gap widths that are absent if $\sigma=0.5 \mathrm{~mm}$. 

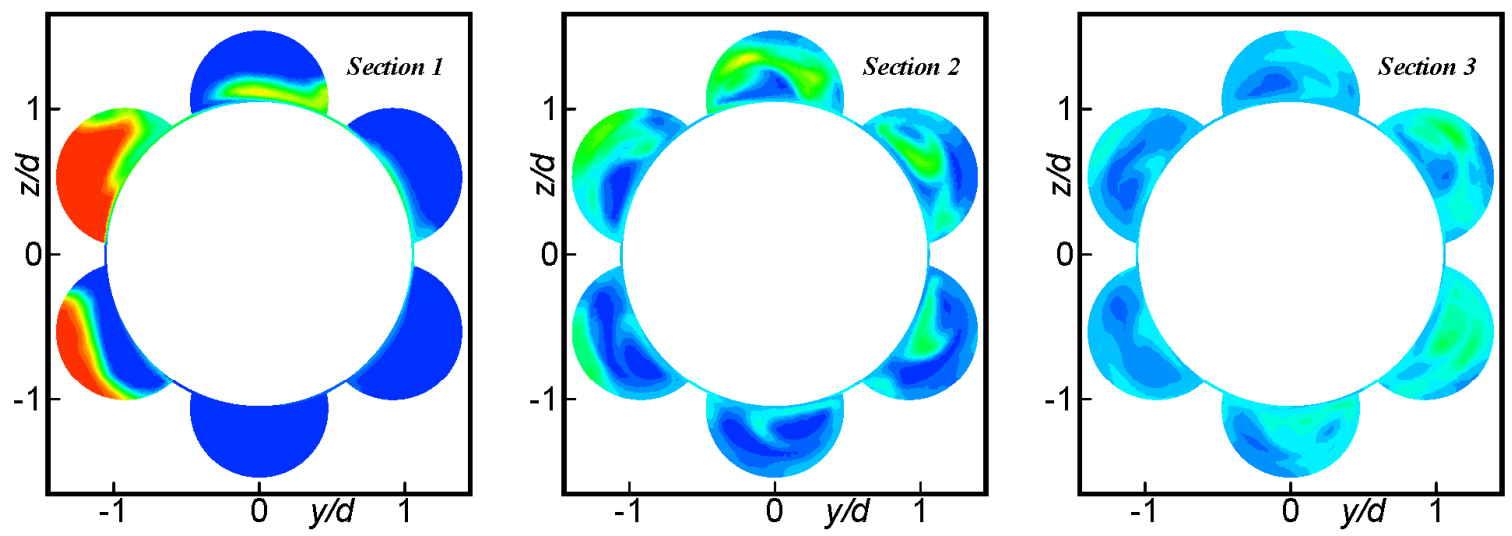

(a)
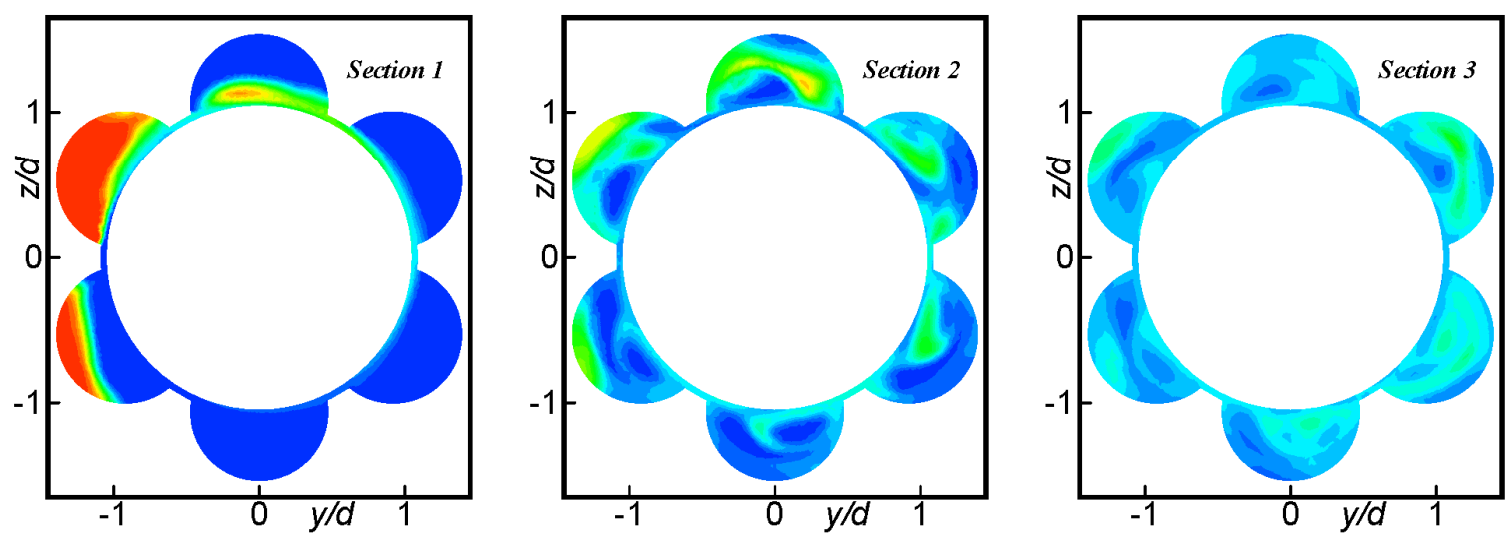

(b)
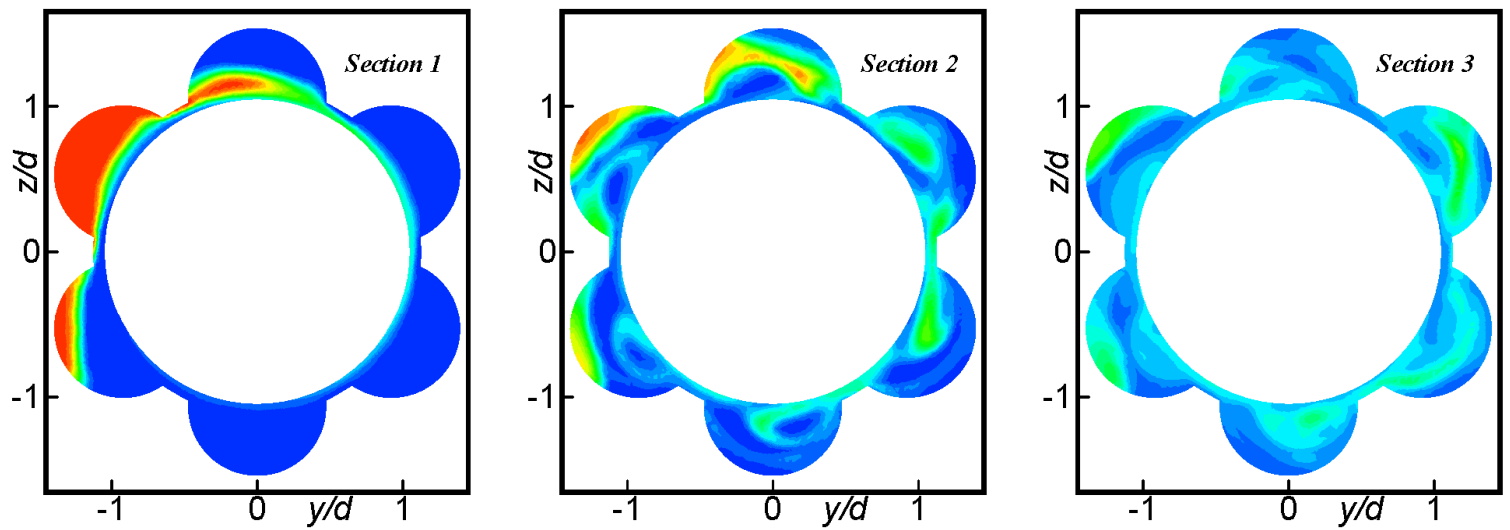

(c)

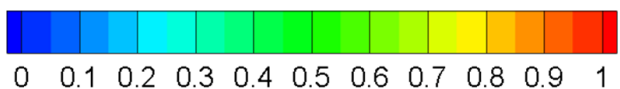

Figure 10. Simulated instantaneous concentration of Liquid 2 with gap width $=0.5 \mathrm{~mm}(\mathrm{a}), 1.5 \mathrm{~mm}(\mathrm{~b})$ and $3.0 \mathrm{~mm}(\mathrm{c})$ in different sections at $t N=10$.

The mixing efficiency of the two miscible high viscosity fluids in the dynamic mixer is quantified in terms of coefficient of variation $(\mathrm{COV})$. It represents the standard deviation of liquid concentration to the 
mean liquid concentration: ${ }^{34}$

$$
\begin{gathered}
\operatorname{COV}=\frac{\left(\frac{1}{A} \iint_{A}\left(C_{a v g}-C_{2}\right)^{2} d A\right)^{0.5}}{C_{\text {avg }}} \\
C_{\mathrm{avg}}=\frac{1}{A} \iint_{A} C_{2} d A
\end{gathered}
$$

where $C_{a v g}$ is the average scalar concentration of the selected section, $\mathrm{C}_{2}$ is the scalar concentration of Liquid 2 and $A$ is the area of the selected section $\left(\mathrm{m}^{2}\right)$.

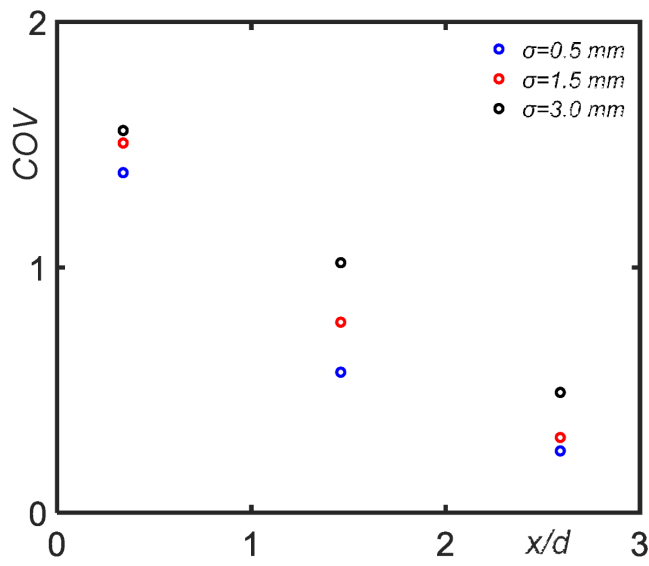

(a)

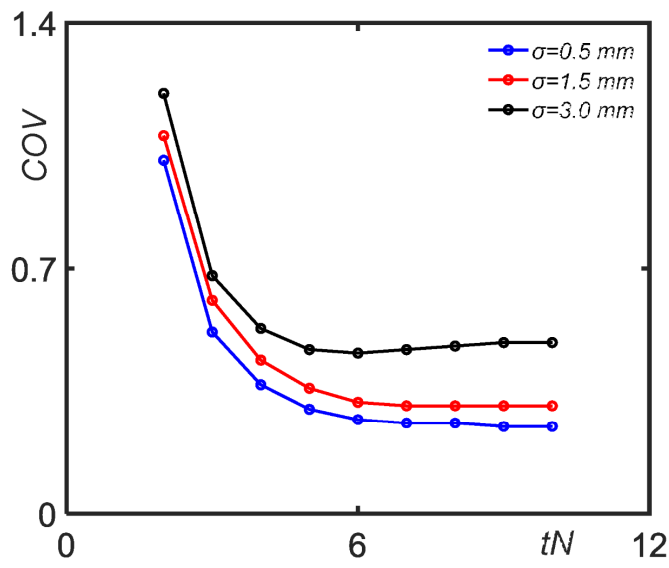

(b)

Figure 11. (a) Simulated coefficient of variation in sections in the dynamic mixer with different gap widths at $t N=10$; (b)

Simulated instantaneous coefficient of variation in Section 3 of the dynamic mixers with different $\sigma$.

In Figure 11(a) $C O V$ of different $y-z$ sections are plotted to quantify the mixing process at $t N=10$. The $C O V$ value decreases from Section 1 to Section 3. In specific $y-z$ sections, the lower the $\sigma$, the smaller the $C O V$ values, and thus the better the liquid mixing. The two fluids are not yet mixed very uniformly in Section 3, which is the final section in our experimental mixer. However, the conventional dynamic mixer will have more cavity rows. For feasibility of the experiments and to minimize the computational cost, there are only three rows of stator cavities in our research. In Figure 11(b), we present time series $C O V$ values with three different $\sigma$ in Section 3. The $C O V$ values decrease with the increase of time at first and then level off, that is, the level of mixing improves with time at first and then remains constant. In all three cases, scalar transport reaches a steady state after approximately 7 rotor revolutions. We saw earlier that 
fluid flow becomes steady within one rotor revolution. The best mixing performance is for $\sigma=0.5 \mathrm{~mm}$. In general, minimizing the gap width is recommended as a way to achieve faster mixing.
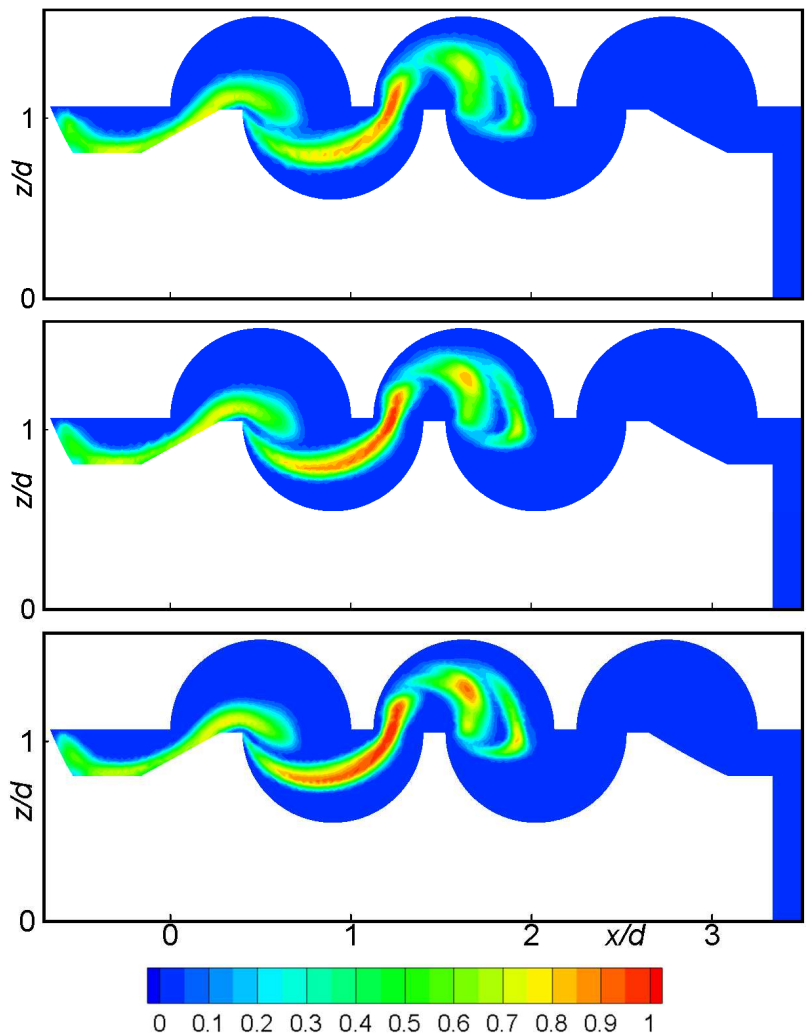

Figure 12. Simulated instantaneous concentration of Liquid 2 in $y / d=0$ plane at $t N=1$ with different grids ( 3.0 million (top), 6.0 million (middle), 10.0 million (bottom)) with $\sigma=0.5 \mathrm{~mm}$.

The simulated instantaneous concentrations of Liquid 2 at $t N=1$ with different grids (3.0 million, 6.0 million and 10.0 million) are shown in Figure 12. Here we see a clear grid dependence, that is, the better mixing is predicted when the number of the cells is lower. This phenomenon was also observed when we simulated the mixing process in a lid-driven cavity. ${ }^{7}$ This is because the scalar diffusion distance $\delta$ over a typical flow time scale (for which we take $d /$ Vtip) is $\delta / d=(S c R e)^{-1 / 2} \approx 3 \times 10^{-4}$. Thus, the number of control volumes in a cavity needs to be $\frac{4}{3} \pi\left(0.5 \times \frac{d}{\delta}\right)^{3}=1.9 \times 10^{10}$ to capture the concentration field in full detail which is computationally unfeasible. Subsequent simulations for identifying trends in scalar mixing are all based on grids with 6.0 million cells, and we realize that grid convergence for the concentration 
field has not yet been reached at this level of resolution. At the same time, we used the Richardson extrapolation $^{28}$ to estimate the asymptotic limit of a characteristic value. We take the $C O V$ value as a characteristic number of the concentration field. The $C O V$ values in the $y=0$ plane at $t N=2$ are $1.64,1.74$ and 1.80 for three different grids (3.6 million, 6.0 million and 10.0 million) respectively. The asymptotic limit $C O V_{\text {lim }}$ value with first-order Richardson extrapolation is 1.89 which is given by:

$$
f_{\text {lim }}=\frac{-f_{2}^{2}+f_{1} f_{3}}{f_{1}-2 f_{2}+f_{3}}
$$

where $f$ is the characteristic number. If we can get a value very close to estimate the asymptotic limit characteristic value with more cells in the grid in future researches, we could say that the simulation has become grid-independent.
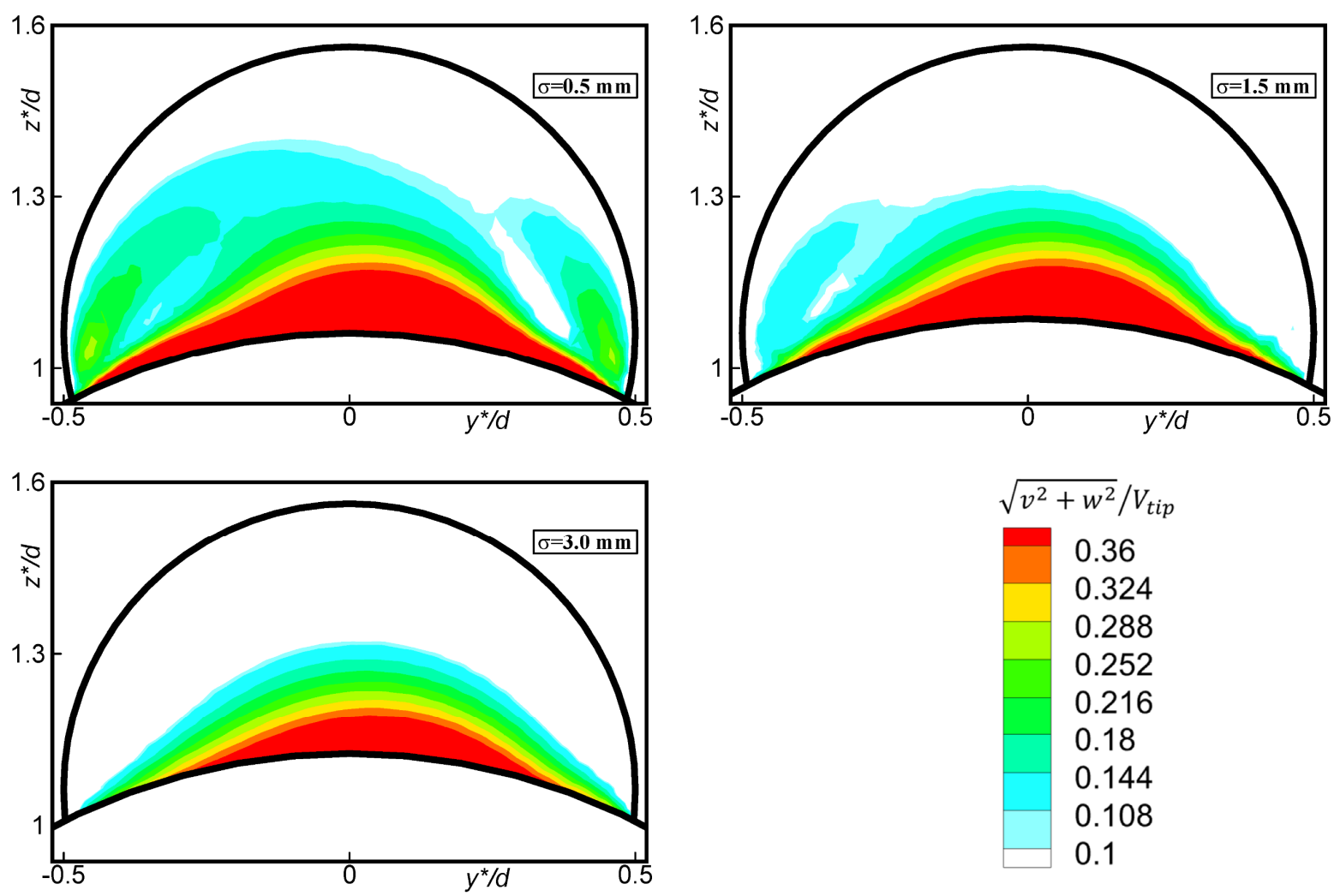

Figure 13. Instantaneous velocity fields in the second stator cavity in Region 2 in Section 3 at $t N=1$ in the dynamic mixers with different gap widths $(\sigma=0.5 \mathrm{~mm}, 1.5 \mathrm{~mm}$ and $3.0 \mathrm{~mm})$. 
To investigate how smaller $\sigma$ can obtain better mixing, the velocity fields in Region 2 at different $\sigma$ are shown in Figure 13. It can be seen that the velocity magnitude fields change significantly with $\sigma$ becomes larger. The area of high-speed near the rotor with velocity magnitude of at least 0.1 Vtip decreases as the $\sigma$ increases. In Figure 14 (a), the velocity $\left(\sqrt{v^{2}+w^{2}} / V_{t i p}\right)$ on the horizontal line $\left(z^{*} / d=1.3\right)$ are chosen to present the quantitative comparisons between cases with different $\sigma$. It is evident that the velocities at $\sigma=3 \mathrm{~mm}$ are generally smaller than at $\sigma=0.5 \mathrm{~mm}$ and $1.5 \mathrm{~mm}$ except the maximum value. As shown in Figure 14 (b), the shear rate increases with the reduction of $\sigma$. The strengthening and weakening of the velocity field and also the shear rate in the rotation direction is a critical factor in how the gap width affects the mixing performance. The pressure drop between the two inlets and outlet and COV in Section 3 at $t N=10$ are shown in Table 1. It can be seen that the pressure drop between the inlets and outlet increases when $\sigma$ decreases. The increased mixing performance with decreasing gap width therefore comes with an increased power consumption.

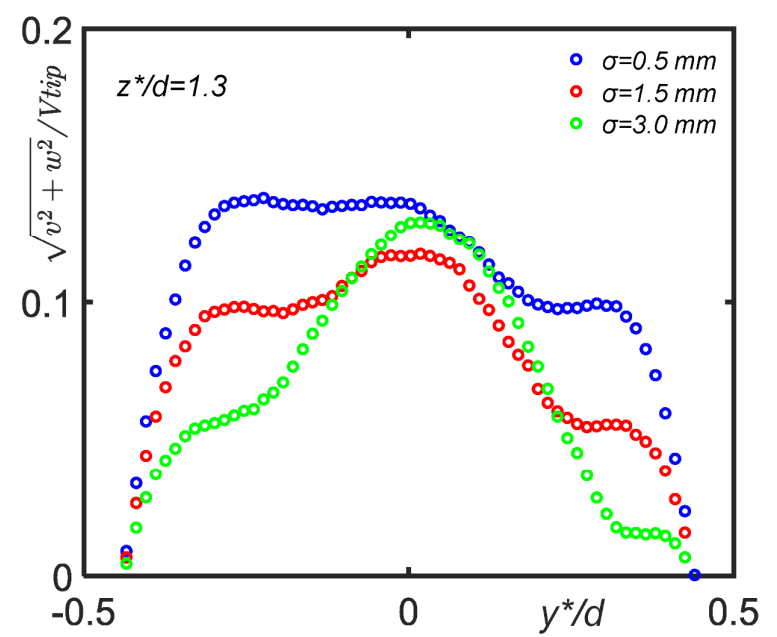

(a)

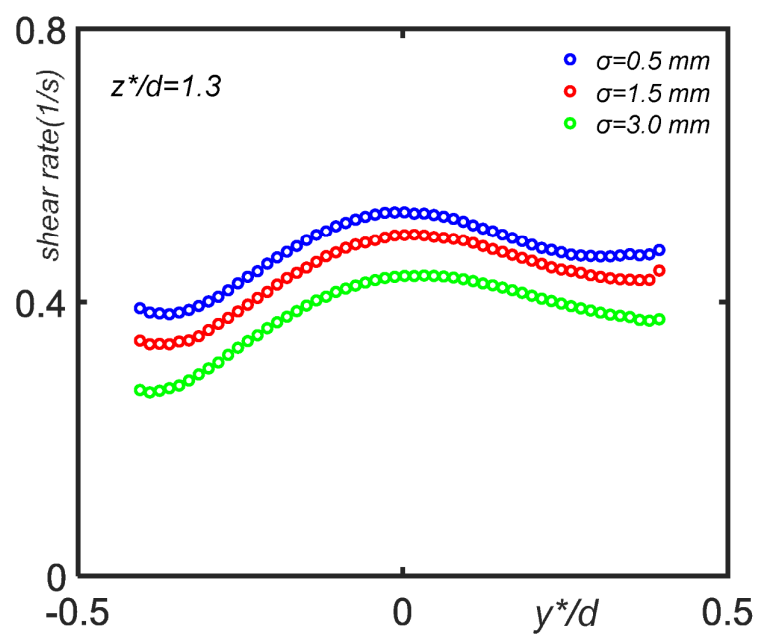

(b)

Figure 14. Instantaneous velocity (a) and shear rate (b) profiles on the horizontal line $\left(z^{*} / d=1.3\right)$ in the second stator cavity in Region 2 at $t N=1$ in the dynamic mixer with different $\sigma$. 
Table 1 The pressure drops between the inlets and outlet $C O V$ in Section 3 at $t N=10$

\begin{tabular}{cccc}
\hline$\sigma$ & $\begin{array}{c}\text { Pressure drop between } \\
\text { Inlet 1 and outlet }(\mathrm{Pa})\end{array}$ & $\begin{array}{c}\text { Pressure drop between } \\
\text { Inlet 2 and outlet }(\mathrm{Pa})\end{array}$ & $\begin{array}{c}\text { COV in Section 3 } \\
\text { at } t N=10\end{array}$ \\
\hline $0.5 \mathrm{~mm}$ & 4410 & 2856 & 0.25 \\
$1.5 \mathrm{~mm}$ & 3950 & 2534 & 0.31 \\
$3.0 \mathrm{~mm}$ & 3477 & 2232 & 0.49 \\
\hline
\end{tabular}

\subsubsection{The effect of $A r / R e$}

In our previous research, ${ }^{7}$ a dimensional group $\frac{A r}{R e}=\frac{\left(\rho_{1}-\rho_{2}\right) g L^{2}}{\mu U}$, which was defined as the ratio of buoyancy $\left(\left(\rho_{1}-\rho_{2}\right) g L^{3}\right)$ to viscous force $\left(\mu \frac{U}{L} L^{2}\right)$, was proposed to characterize the mixing process of two miscible fluids in a lid-driven cavity. To investigate the effect of $A r / R e$ on the mixing performance in the dynamic mixer, two pairs of simulations with the same $A r / R e$ were conducted. The parameters of these cases are shown in Table 2.

Table 2 Parameters of two pairs with the same value of $\frac{A r}{R e}$

\begin{tabular}{|c|c|c|c|c|c|c|c|}
\hline Pairs & $\begin{array}{c}\rho_{1} \\
\left(\mathrm{~kg} / \mathrm{m}^{3}\right)\end{array}$ & $\begin{array}{c}\rho_{2} \\
\left(\mathrm{~kg} / \mathrm{m}^{3}\right)\end{array}$ & $\begin{array}{l}\mu_{1}=\mu_{2} \\
(\mathrm{~Pa} \cdot \mathrm{s})\end{array}$ & $\begin{array}{l}\text { Volume rate of Inlet } 1 \\
\qquad(\mathrm{ml} / \mathrm{min})\end{array}$ & $\begin{array}{l}\text { Volume rate of Inlet } 2 \\
\qquad(\mathrm{ml} / \mathrm{min})\end{array}$ & $\begin{array}{l}\text { Gap width, } \sigma \\
(\mathrm{mm})\end{array}$ & Ar/Re \\
\hline$a_{1}$ & 1394 & 1386 & 10 & 192 & 48 & 0.5 & 12.6 \\
\hline$a_{2}$ & 1394 & 1390 & 5 & 192 & 48 & 0.5 & 12.6 \\
\hline$b_{1}$ & 1394 & 1294 & 1 & 192 & 48 & 0.5 & 1575 \\
\hline$b_{2}$ & 1394 & 1244 & 0.5 & 192 & 48 & 0.5 & 1575 \\
\hline
\end{tabular}

Instantaneous concentration distribution at $t N=10$ in the $y / d=0$ plane as well as in Section 3 are shown in Figure 15. These two pairs of simulations with various density difference and viscosity but the same $\frac{A r}{R e}$ almost have the same concentration distribution in different planes. In Figure 16, time-series of $C O V$ are plotted to quantify the mixing process in Section 3. The almost overlapping $C O V$ curves confirm that cases having the same $\frac{A r}{R e}$ value will lead to the same concentration distribution and mixing performance. 
Better mixing performance can be achieved with small $\frac{A r}{R e}$ (compare results for $\frac{A r}{R e}=12.6$ and $\frac{A r}{R e}=1575$ ).

(a1) $\frac{A r}{R e}=12.6, R e=0.0164$
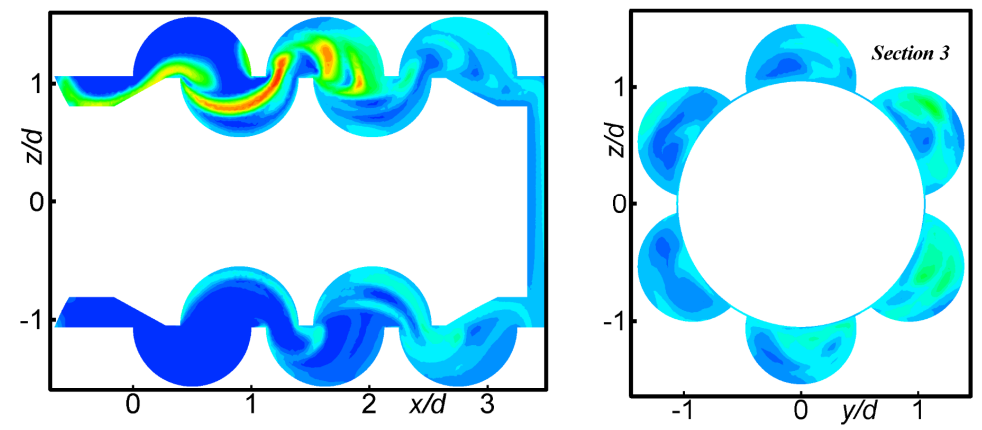

(a) $\frac{A r}{R e}=12.6, R e=0.0328$
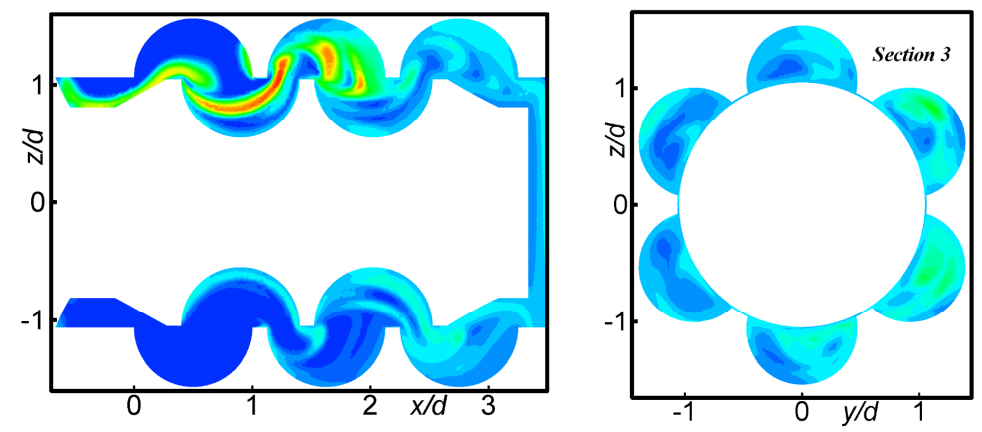

(b) $\frac{A r}{R e}=1575, R e=0.164$
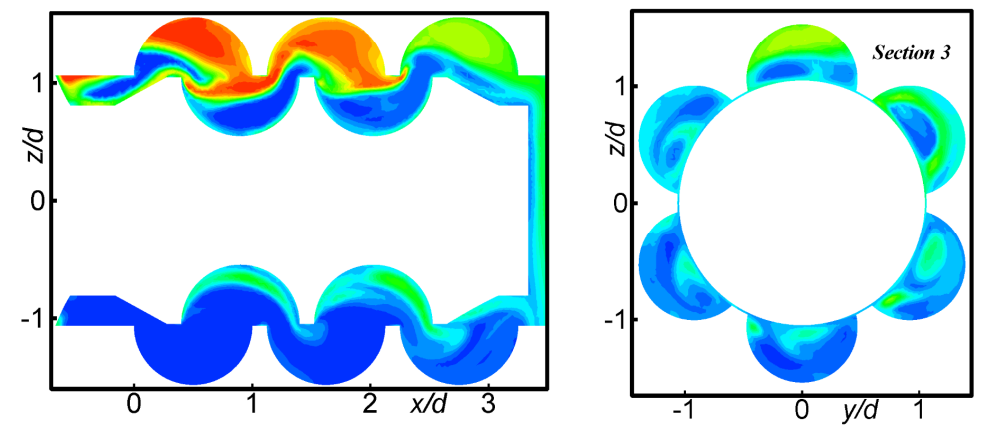

(b2) $\frac{A r}{R e}=1575, R e=0.328$
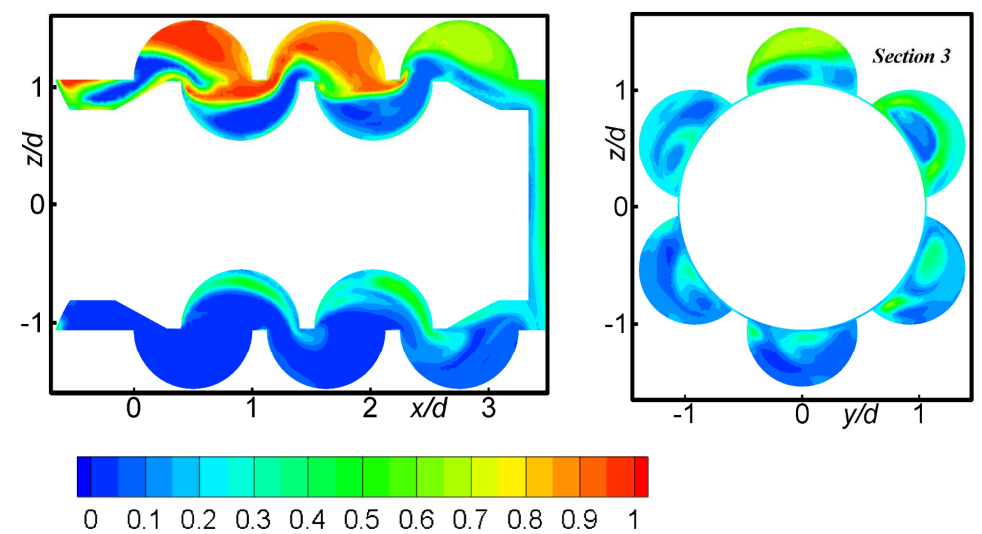

Figure 15. Two pairs of simulated instantaneous concentration of Liquid 2 in the $y / d=0$ plane (left column) and Section 3 (right column), each pair of cases have same $\frac{A r}{R e}$ but different $R e$. (Detailed parameters of a1, a2, b1 and b2 see Table 2) 


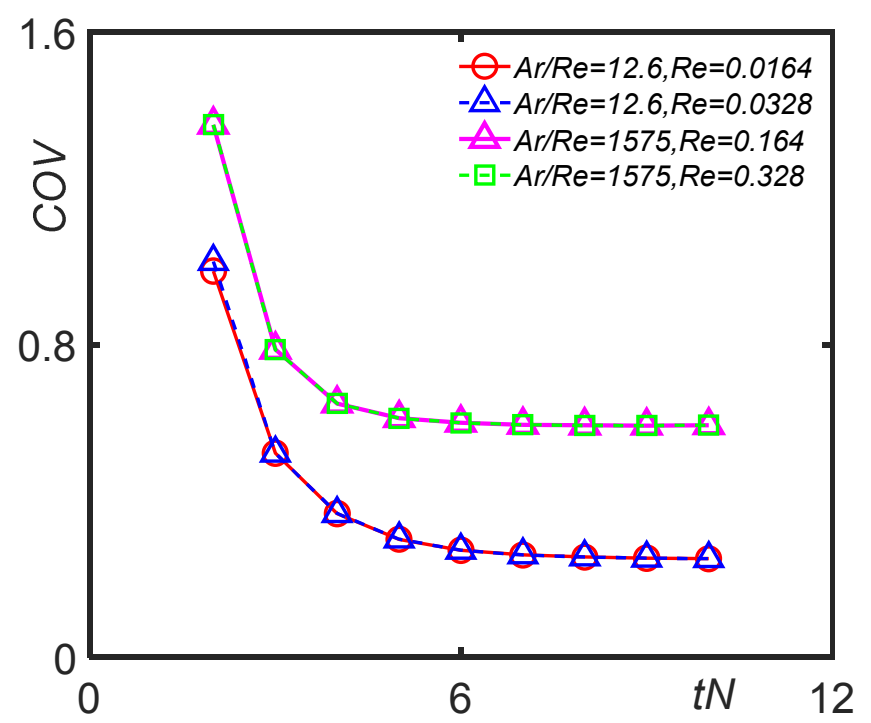

Figure 16. Two pairs of simulated instantaneous coefficient of variation in Section 3 with same Ar/Re but different $R$ e.

\section{Conclusions and future work}

A simplified industrial dynamic mixer was investigated in this work. Refractive index matching between the working fluids and the test section of the dynamic mixer was achieved in order to be able to visualize the flow fields. Average flow fields in two representative regions of the dynamic mixer were measured by using the 2D-PIV experiments. To the best of our knowledge, it is the first work on visualizing the flow fields of high viscosity fluids in a dynamic mixer by using PIV experiments. This work demonstrates the feasibility of highly resolved flow velocity measurements of high-viscosity fluids in different regions of such mixers that have a complex dynamic geometry.

CFD simulations with species transport models were used to predict the velocity fields and instantaneous concentration distribution in the dynamic mixer. The simulated velocity fields in two typical regions are validated quantitatively by the experimental results, and they agree very well with each other. On this basis, various flow patterns along flow and rotation directions in the dynamic mixer are discussed.

The effects of the gap width between rotor and stator on scalar mixing have been discussed. The mixing performance becomes better with the decrease of $\sigma$ because of the enhanced shearing and more 
energy consumption in terms of the $C O V$ of fluid concentration. A dimensionless group $\frac{A r}{R e}$ is applied to investigate the effect of fluids properties such as density difference and viscosity on scalar mixing. At

constant $\frac{A r}{R e}$ value, we obtain approximately the same mixing performance and concentration distribution. At the same time, better mixing performance can be achieved with smaller $\frac{A r}{R e}$.

In this work, we created a RI matched PIV system with PMMA and a maltose aqueous solution for the dynamic mixer and the working fluid respectively. We investigated the flow characteristics in the dynamic mixer based on the RI matched PIV experiments and validated computational methods and models. The flow and mixing characteristics in the dynamic mixer have been discussed, although the grid effects on the concentration field are difficult to avoid because of the high Schmidt number. In the future, we will experimentally and numerically focus on the mixing process of miscible non-Newtonian fluids with a wide range of Reynolds numbers not only in the cavity transfer dynamic mixer but also in other mixers with complex dynamic geometry.

\section{Author Information}

Corresponding Authors

*E-mail lizp@mail.buct.edu.cn (Zhipeng Li).

*E-mail gaozm@mail.buct.edu.cn (Zhengming Gao)

\section{Acknowledgment}

The authors appreciatively acknowledge the financial support from the National Key Research and Development Program of China (No.2016YFB0302801), National Natural Science Foundation of China (No.21676007), the Fundamental Research Funds for the Central Universities (XK1802-1) and the China Scholarship Council. 


\section{References}

(1) Zhang, C.; Ferrell, A. R.; Nandakumar, K. Study of a toroidal-helical pipe as an innovative static mixer in laminar flows. Chem. Eng. J. 2019, 359, 446-458.

(2) Wittek, P.; Pereira, G. G.; Emin, M. A.; Lemiale, V. Cleary PW. Accuracy analysis of SPH for flow in a model extruder with a kneading element. Chem. Eng. Sci. 2018, 187, 256-268.

(3) Camesasca, M.; Manas-Zloczower, I.; Kaufman, M. Influence of extruder geometry on laminar mixing: entropic analysis. Plast., Rubber Compos. 2004, 33, 372-376.

(4) Ottino, J. M.; Chella, R. Laminar mixing of polymeric liquids; a brief review and recent theoretical developments. Polym. Eng. Sci. 1983, 23, 357-379.

(5) Hindmarch, R. S. The cavity transfer mixer: a blender for all seasonings. Mater. Des. 1987, 8, 331-339.

(6) Jiang, Z.; Guo, Z.; Jia, Z.; Pu, C.; Xiao, C.; Jin, J. Mixing mechanism and evaluation of the dispersion effect of a 3D dynamic Mixer. J. Test. Eval. 2016, 44, 367-375.

(7) Huang, F.; Wang, D.; Li, Z.; Gao, Z.; Derksen, J. J. Mixing process of two miscible fluids in a lid-driven cavity. Chem. Eng. J. 2019, 362, 229-242.

(8) Wang, C.; Manas $\square$ Zloczower, I. Flow field analysis of a cavity transfer mixer. Polym. Eng. Sci. 1994, 34, 1224-1230.

(9) Grosso, G.; Hulsen, M. A.; Fard, A. S.; Anderson, P. D.; Overend, A. Mixing processes in the cavity transfer mixer: a thorough study. AIChE J. 2018, 64, 1034-1048.

(10) Woering, A. A.; Gorissen, W. C. M.; Biesheuvel, A. Optimization of viscous mixing in a two-dimensional cavity transfer mixer. Flow. Turbul. Combust. 1998, 60, 377-407.

(11) Grosso, G.; Hulsen, M. A.; Overend, A.; Anderson, P. D. Fluid flow and distributive mixing analysis in the cavity transfer mixer. Macromol. Theory Simul. 2018, 27, 1700075. 
(12) Lester, D. R.; Rudman, M.; Metcalfe, G. Low Reynolds number scalar transport enhancement in viscous and non-Newtonian fluids. Int J Heat Mass Transf. 2009, 52, 655-664.

(13) Voth, G. A.; Saint, T. C.; Doble, G.; Gollub, J. P. Mixing rates and symmetry breaking in two-dimensional chaotic flow. Phys Fluids. 2003, 15, 2560-2566.

(14) González-Neria, I.; Alonzo-Garcia, A.; Martínez-Delgadillo, S. A.; Mendoza-Escamilla, V. X.; Yáñez-Varela, J. A.; Verdin, P. G.; Rivadeneyra-Romero, G. PIV and dynamic LES of the turbulent stream and mixing induced by a V-grooved blade axial agitator. Chem. Eng. J. 2019, 374, 1138-1152.

(15) Hoffmann, M.; Schlüter, M.; Räbiger, N. Experimental investigation of liquid-liquid mixing in T-shaped micro-mixers using $\mu$-LIF and $\mu$-PIV. Chem. Eng. Sci. 2006, 61, 2968-2976.

(16) Li, G.; Gao, Z.; Li, Z.; Wang, J.; Derksen, J. J. Particle-resolved PIV experiments of solid-liquid mixing in a turbulent stirred tank. AIChE J. 2018, 64, 389-402.

(17) Li, G.; Li, Z.; Gao, Z.; Wang, J.; Bao, Y.; Derksen, J. J. Particle image velocimetry experiments and direct numerical simulations of solids suspension in transitional stirred tank flow. Chem. Eng. Sci. 2018, 191, 288-299.

(18) Gong, H.; Huang, F.; Li, Z.; Gao, Z.; Derksen, J. J. Mechanisms for drawdown of floating particles in a laminar stirred tank flow. Chem. Eng. J. 2018, 346, 340-350.

(19) Wiederseiner, S.; Andreini, N.; Epely-Chauvin, G.; Ancey, C. Refractive-index and density matching in concentrated particle suspensions: a review. Exp. Fluids 2011, 50, 1183-1206.

(20) Yuki, K.; Hasegawa, S.; Sato, T.; Hashizume, H.; Aizawa, K.; Yamano, H. Matched refractive-index PIV visualization of complex flow structure in a three-dimensionally connected dual elbow. Nucl. Eng. Des. 2011, 241, 4544-4550.

(21) Mazhar, H.; Ewing, D.; Cotton, J. S.; Ching, C. Y. Measurement of the flow field characteristics in 
single and dual S-shape $90^{\circ}$ bends using matched refractive index PIV. Exp. Therm. Fluid Sci. 2016, 79, $65-73$.

(22) Jaffer, S. A.; Bravo, V. L.; Wood, P. E.; Hrymak, A. N.; Wright, J. D. Experimental validation of numerical simulations of the kneading disc section in a twin screw extruder. Polym. Eng. Sci. 2010, 40, 892-901.

(23) Zhu, L.; Cai, T.; Huang, J.; Stringfellow, T. C.; Wall, M.; Yu, L. Water self-diffusion in glassy and liquid maltose measured by Raman microscopy and NMR. J. Phys. Chem. B 2011, 115, 5849-5855.

(24) Zhang, Y.; Gao, Z.; Li, Z.; Derksen, J. J. Transitional flow in a Rushton turbine stirred tank. AIChE J. 2017, 63, 3610-3623.

(25) Liang, Y.; Shi, D.; Xu, B.; Cai, Z.; Gao, Z. Turbulent flow field in a stirred vessel agitated by an impeller with flexible blades. AIChE J. 2018, 64, 4148-4161.

(26) Dahikar, S. K.; Joshi, J. B.; Shah, M. S.; Kalsi, A. S.; RamaPrasad, C. S.; Shukla, D. S. Experimental and computational fluid dynamic study of reacting gas jet in liquid: flow pattern and heat transfer. Chem. Eng. Sci. 2010, 65, 827-849.

(27) Tam, A.; Ait-Ali-Yahia, D.; Robichaud, M. P.; Moore, M.; Kozel, V.; Habashi, W. G. Anisotropic mesh adaptation for 3D flows on structured and unstructured grids. Comput Methods Appl Mech Eng. 2000, $189,1205-1230$.

(28) Ferziger, J.H.; Perić, M. Computational methods for fluid dynamics (3rd edition); Springer: Berlin, 2002; pp 58-156.

(29) Leer, B. V. Towards the ultimate conservative difference scheme. V. A second-order sequel to Godunov's method. J Comput Phys. 1979, 32, 101-136.

(30) Hamidipour, M.; Chen, J.; Larachi, F. CFD study and experimental validation of trickle bed 
hydrodynamics under gas, liquid and gas/liquid alternating cyclic operations. Chem. Eng. Sci. 2013, 89, $158-170$.

(31) Liu, M. Computational study of convective-diffusive mixing in a microchannel mixer. Chem. Eng. Sci. 2011, 66, 2211-2223.

(32) Liberzon, A. On the effects of dilute polymers on driven cavity turbulent flows. Int. J. Heat Fluid Flow. 2011, 32, 1129-1137.

(33) Anupindi, K.; Lai, W.; Frankel, S. Characterization of oscillatory instability in lid driven cavity flows using lattice boltzmann method. Comput. Fluids. 2014, 92, 7-21.

(34) Mandal, M. M.; Aggarwal, P.; Nigam, K. D. P. Liquid-liquid mixing in coiled flow inverter. Ind. Eng. Chem. Res. 2011, 50, 13230-13235. 
For Table of Contents Only
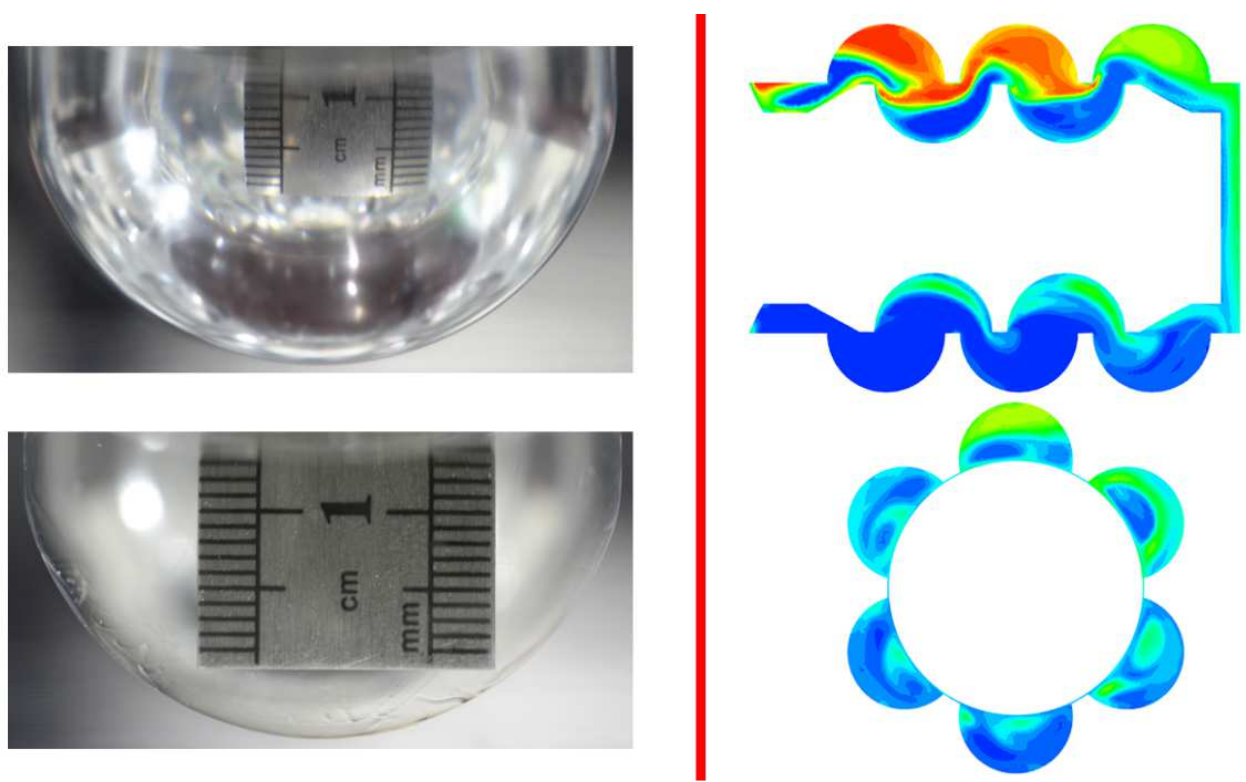